\title{
A Comparative Study of Braided versus Barbed Suture for Cystotomy Repair
}

\author{
Robert Shapiro $\mathbb{D}^{1,2}$ \\ Alec Sunyecz $\mathbb{D D}^{3}$ \\ Stanley Zaslau ${ }^{1,2}$ \\ Manuel C Vallejo ${ }^{1,4}$ \\ Tyler Trump ${ }^{2}$ \\ Omar Dueñas-Garcia' \\ 'Department of Obstetrics and \\ Gynecology, West Virginia University \\ School of Medicine, Morgantown, WV, \\ 26506, USA; ${ }^{2}$ Department of Urology, \\ West Virginia University School of \\ Medicine, Morgantown, WV, 26506, USA; \\ ${ }^{3}$ West Virginia University School of \\ Medicine, Morgantown, WV, 26506, USA; \\ ${ }^{4}$ Department of Anesthesiology, West \\ Virginia University School of Medicine, \\ Morgantown, WV, 26506, USA
}

Background: In this study, we aim to compare outcomes after cystotomy repair between standard sutures (910 polyglactin, poliglecaprone) versus barbed (V-Loc ${ }^{\mathrm{TM}}$ 90) suture. As a secondary outcome, we analyzed factors for suture preference between the two groups.

Methods: A retrospective chart review was undertaken for surgeries complicated by cystotomy, identified by ICD-9/10 codes from 2016 to 2019 at West Virginia University (WVU) Hospital. Comparisons were made between cystotomy repair using barbed suture versus standard braided suture. Injuries were categorized by procedure, surgical route, type of suture used in repair, and subsequent complications related to repair. Primary endpoints were examined by Pearson's Chi-square test and interval data by $t$-test. A $\mathrm{p}<0.05$ was significant. Results: Sixty-eight patients were identified with iatrogenic cystotomy at WVU. Barbed suture was used for cystotomy repair in 11/68 (16.2\%) patients. No significant difference was seen in postoperative outcomes between patients repaired with barbed suture versus standard braided suture. Barbed suture was significantly more likely to be used for cystotomy repair in minimally invasive surgery $(\mathrm{p}=0.001)$. It was most often utilized in a robotic approach $7 / 11$ (63.6\%) followed by laparoscopic 3/11 (27.3\%). Body mass index (BMI) was significantly higher in patients receiving a barbed suture repair $(\mathrm{p}=0.005)$.

Conclusion: Barbed suture may be comparable to standard braided suture for cystotomy repair. Barbed suture may offer a practical alternative to facilitate cystotomy repair in minimally invasive surgery, especially in patients with a high BMI.

Keywords: barbed suture, cystotomy, minimally invasive surgery, obesity

\section{Background}

Urinary tract injuries are an unwanted complication of any pelvic surgery. It has been shown that urinary tract injuries occur in $0.2-1 \%$ of all pelvic operations especially in those with adhesions or distorted anatomy. ${ }^{1}$ During pelvic surgeries, injuries are more likely to involve the bladder, followed by the ureter. ${ }^{2-4}$

Many cystotomy repair techniques have been studied including the mechanism and instruments needed for repair. The traditional closure of an iatrogenic bladder laceration requires intraoperative repair in two layers using absorbable sutures followed by back-filling the bladder to observe no urinary leakage. ${ }^{5,6}$ Achieving this laparoscopically can be difficult, requiring many hours of practice to gain proficiency. Figert et al concluded from their study that specific training and experience are needed to develop such laparoscopic skills. ${ }^{7}$

Laparoscopic surgery within the pelvis can present multiple challenges, especially when a urologic injury is encountered. Some of these challenges include limited visualization, excess fat distribution, redundant colon, and malpositioning
Correspondence: Robert Shapiro Health Science Center North, West Virginia University, PO Box $9001 \mathrm{~A}$ Morgantown, WV, 26506, USA

Tel $+\mid$ 304-293-563|

Fax + 304-293-5I60

Email rshapiro@hsc.wvu.edu 
of instruments. ${ }^{8}$ To avoid opening the patient to facilitate a repair of a urologic injury, techniques to mitigate these challenges must be implemented.

Barbed suture, such as the V-Loc 90, was first used in urologic procedures in 2008 and demonstrates many advantages over conventional laparoscopic suturing and knot tying. ${ }^{9}$ Barbed suture has been shown to shorten laparoscopic training time, reduce operating room cost, increase safety, and potentially improve outcomes in minimally invasive surgery. 9,10 The design of the barbed sutures incorporates unidirectional or bidirectional barbs allowing for tension to be distributed evenly along the suture line. Barbed sutures were observed to be superior to Monocryl and Vicryl in a rabbit bladder model study. ${ }^{11}$ In another study using porcine bladders, it was found that barbed sutures significantly reduced operative time while providing easier tissue approximation. ${ }^{11}$

In this study, we aim to compare outcomes of traditional suture repair of urologic injury during open surgery to laparoscopic repair using a barbed V-Loc suture. As a secondary outcome, we analyzed factors for suture preference between the two groups.

\section{Methods}

This is a retrospective cohort study using the medical records from West Virginia University Hospital. IRB approval for this study was acquired by the West Virginia University Institutional Review Board (approval number 1909723019). The study was conducted according to the criteria set by the declaration of Helsinki. In this study, we identified the patients based on a query of electronic medical records using current procedural terminology codes and ICD-9 diagnosis. The search was performed from January 2016 - September 2019. The study was limited from January 2016 as barbed suture was not readily available for use at West Virginia University Hospital until this date. For all bladder repairs, we used the CPT codes: 51,860 cystorrhaphy, suture of bladder wound, injury or rupture and 51,040 incision procedures on the bladder. Patient charts were retrospectively reviewed by A.S. and information was obtained on basic demographics and surgical history. Injuries to the bladder were recorded along with type of surgery (gynecologic, urologic, other), manner of surgery (open, laparoscopic, robotic), total operative time, post-operative studies (fluoroscopy, voiding trial), time to Foley removal, length of hospital stay, and mechanism of repair (open, laparoscopic, robotic). Total operative time included the length of the primary procedure with bladder repair. All bladder injury repairs were evaluated by recording the suture material that was used (Vicryl TM, r Barbed V-Loc TM). Vicryl suture is a braided absorbable suture made from 90:10 poly(glycolide-co-L(-)-lactide). Barbed V-Loc suture is a synthetic absorbable unidirectional barbed suture prepared from a synthetic polyester composed of glycolide, dioxanone, and trimethylene carbonate. No surgical knots were tied when this suture was used.

Vicryl suture is traditionally used in bladder repair, and their use is based on surgeon preference. All patients underwent a standard general anesthetic. Any complications in subsequent patient encounters were also recorded. Complications were defined as any urinary tract diagnoses that developed following the recorded surgical procedure. Statistical analysis was performed with Pearson's ChiSquare test of association and interval data by $t$-test. A p $<0.05$ was significant. Data were analyzed using JMP software Version Pro 12.2, SAS Institute Inc., Copyright 2018.

\section{Results}

Our review incorporated 68 patients with a mean age of $52.2 \pm 20.1$ years. There was an almost equal representation of gender with 35 males $(51.5 \%)$ and 33 females (48.5\%). BMI is an important consideration in surgery especially with respect to technical difficulty and potential for complications. While the mean BMI of our study was technically overweight $(27.5 \pm 7.9)$, a significant portion of patients fell into the normal (39.7\%) and obese (33.8\%) categories based on BMI. The complete demographic breakdown is summarized in Table 1. No differences were noted with respect to operative time, post-operative fluoroscopy (if ordered), time to Foley removal (if placed),

Table I Patient Demographic Characteristics

\begin{tabular}{|l|l|c|c|}
\hline Characteristics & Mean & Barbed & Braided \\
\hline Age & $52.2 \pm 20.1$ & 42 & 54 \\
\hline Gender & Female: $33(48.5 \%)$ & 8 & 25 \\
& Male: 35 (5I.5\%) & 3 & 32 \\
\hline Race & White: N=67 & 11 & 56 \\
& Asian N= I & & I \\
\hline BMI & $27.5 \pm 7.9$ & $33.6 \pm 13$ & $26.3 \pm 6$ \\
\hline
\end{tabular}

Note: Patient demographics included in the study were separated into age, gender, race, and BMI. 
Table 2 Cystotomy Repair Results

\begin{tabular}{|l|l|l|}
\hline & Barbed & Braided \\
\hline Laparoscopic Repair & $3^{*}$ & 8 \\
Open Repair & $\mathrm{I}^{\pi}$ & 18 \\
Robotic Repair & $7^{*}$ & 4 \\
Operative Time (min) & $162.0 \pm 84.5$ & $244.7 \pm 144.7$ \\
Time to Foley Removal (days) & $17.1 \pm 12.4$ & $14.5 \pm 7.2$ \\
Length of Stay (days) & $3.5 \pm 3.9$ & $6.1 \pm 5.4$ \\
Post Operative Fluroscopy & 9 & 23 \\
Post Operative Voiding Trial & 1 & 2 \\
Complications & 3 & 8 \\
\hline
\end{tabular}

Notes: ${ }^{*} p<0.05 ; \pi_{p}<0.01$. The results display the setting of cystotomy repair and patient outcomes between barbed and non barbed suture.

and hospital length of stay. These results are listed in Table 2.

Barbed suture was used for cystotomy repair in 11/68 (16.2\%) patients, and the mean age of this group was 42.2 \pm 20.0 years. In the braided suture group, the mean age was older at $54.0 \pm 19.5$ years. Age between suture groups was not a significant finding $(\mathrm{p}=0.0725)$. The mean BMI in the barbed suture group was $33.6 \pm 12.6$ and was significantly higher than the braided suture group $(26.4 \pm 6.1)$ ( $\mathrm{p}=0.0046$, Table 1). Additionally, there was not a significant gender difference between the two suture groups $(\mathrm{p}=0.3029$, Table 1$)$.

Most of the patients had a previous surgical history 41/ $68(60.3 \%)$. Twenty-one patients had a history of open surgery, and 27 patients had a previous surgery that was completed laparoscopically. Of the patients with a history of laparoscopic surgery, 9 were gynecologic related surgeries. Ten patients had a history of cesarean section. There was no statistically significant difference when comparing surgical history and type of suture used in subsequent cystotomy repair $(p=0.1703)$. We explored the use of suture types for cystotomy repair relative to obstetric history. Of the female patients, 19/33 (57.6\%) were previously pregnant. Three of the patients in which barbed suture was used were previously pregnant; however, the type of suture used was non-significant based on gravida status $(\mathrm{p}=0.2113)$. Similarly, there was no difference between suture use and a birth history $(p=0.2801)$.

In our review, cystotomy repair was completed in a variety of settings. Since gynecologic procedures make up a large proportion of urinary tract injuries, ${ }^{2,3}$ female patients were categorized based on whether the primary procedure was hysterectomy $12 / 33(36.3 \%)$ or nonhysterectomy $21 / 33(67.5 \%)$. Of the gynecologic group, barbed suture was used in 5 patients. Of the nongynecologic group, barbed suture was used in 3 patients. The type of suture selected for cystotomy repair relative to the procedure type was not significant $(p=0.6010)$. Within the male patients, barbed suture was used for cystotomy repair in 2 iatrogenic injuries during prostatectomies and 1 trauma injury. The iatrogenic injuries were repaired robotically. The trauma injury was repaired open.

Groups were also categorized based on whether cystotomy repair was performed in a laparoscopic 11/41 (26.8\%), robotic $11 / 41(26.8 \%)$ or open $19 / 41(46.4 \%)$ setting. When barbed suture was used for cystotomy repair, it was most often utilized in a robotic setting $7 / 11$ $(63.6 \%)$ followed by laparoscopic $3 / 11(27.3 \%)$ then open $1 / 11(9.1 \%)$. The use of barbed suture relative to each mode of surgery was significant when compared to braided suture use. A summary of these results is listed in Table 2.

There were 11 complications noted. Complications were defined as subsequent bladder conditions that developed after cystotomy repair requiring additional follow-up or treatment. The overall complication rate in our study was 0.16 . Three were associated with barbed suture use, but this was not statistically significant from the complication rate of braided suture use $(\mathrm{p}=0.9842)$. Complications were further stratified according to Clavien-Dindo Classification $^{12}$ (Table 3 ). The complications were comparable between barbed and braided suture. Most of the complications were grade 1 (Barbed: $\mathrm{N}=2 / 3,67 \%$ ) (Braided: $\mathrm{N}=7 / 8,88 \%$ ).

\section{Discussion}

There are multiple challenges that make minimally invasive knot tying a difficult skill to master. The loss of depth perception, the limited degree of movement, and loss of

Table 3 Complications Stratified According to Clavien-Dindo Classification

\begin{tabular}{|l|l|l|}
\hline & Barbed $(\mathbf{n}=\mathbf{3})$ & Braided $(\mathbf{n}=8)$ \\
\hline I & $2(67 \%)$ & $7(88 \%)$ \\
II & 0 & 0 \\
IIIa & 0 & I (I2\%) \\
IIIb & I (33\%) & 0 \\
IV & 0 & 0 \\
V & 0 & 0 \\
\hline
\end{tabular}

Notes: I: Any deviation from the normal postoperative course without the need for straightforward pharmacological treatment, surgical, or radiologic intervention. II: Requiring pharmacological treatment. III: Requiring surgical or radiologic intervention IIla: Intervention not under general anesthesia IIlb: Intervention under general anesthesia IV: Life-threatening complication V: Death of a patient. 
tactile feedback are obstacles that must be overcome to develop competency in a laparoscopic setting. Moreover, proficient knot tying in open surgery does not translate to laparoscopic knot tying ability and requires consistent practice. ${ }^{7}$ Robotic surgery maintains depth perception and increases the degree of operative movement; however, the loss of immediate feedback when suturing by hand in an open case may result in "air" knots or knot overtightening leading to tissue ischemia., 9,13

With barbed suture, knot tying is not necessary. This reduces the skill required to use it. The barbs of the suture self-anchor to provide consistent tissue apposition and eliminate the necessity of an additional hand to follow the suture. Without the need for knots in barbed suture, the risk of suture failure is decreased. ${ }^{14}$

Ultimately, the technical aspects of suturing with laparoscopic instruments are eliminated with barbed suture. Therefore, we would expect a greater use in minimally invasive surgery as compared to open surgery. We saw a statistically significant use of barbed suture in laparoscopic vs open, robotic vs open, and laparoscopic vs robotic procedures over non-barbed suture. Although the sample size is limited, this data supports the utility of barbed suture in a minimally invasive approach to cystotomy repair.

A large portion of our study population was overweight or obese. Obesity is a rapidly growing problem, especially in the United States, and these patients tend to have more concurrent comorbidities. ${ }^{8}$ In addition, operating on patients with an elevated BMI adds complexity. To improve outcomes in these patients, the least invasive approach should be offered. With preparation and optimization of comorbidities, minimally invasive surgery is the safest approach with the lowest risk of complications. ${ }^{8,15,16}$ Although more challenging in obese patients, an experienced anesthetic team, proper set-up and entry can increase chances of a successful minimally invasive procedure. Unexpected complications like injury to the bladder may arise due to pelvic adhesions, limited visibility, and anatomical distortion. ${ }^{1}$ Immediate repair can be done if an injury is recognized intraoperatively. Typically, when an injury occurs during a laparoscopic procedure, the repair is optimally completed laparoscopically to avoid laparotomy. ${ }^{2,3}$ However, a traditional bladder repair requires precise suturing and knot tying to prevent long-term sequelae. Achieving this laparoscopically is among the most difficult skills to master, requiring much practice to gain proficiency. The knotless barbed suture has been proposed to make laparoscopic suturing easier and more efficient. Several in vivo studies have demonstrated faster closure times and reduction of difficulty with the use of barbed suture. ${ }^{13,17}$ Angioli et al assessed barbed suture use in laparoscopic myomectomy and found a significant decrease in suturing time and blood loss in the barbed suture group. ${ }^{18}$ Although a trend towards a shorter operative time was noted in our barbed suture group, this did not reach statistical significance. Also, exact suturing time of the bladder repair was not able to obtain on account of the retrospective study design. We found a significantly higher BMI in the patients that had cystotomy repair utilizing barbed suture. We also found greater barbed suture utilization in a minimally invasive setting. Our study suggests that the advantages of barbed suture make it suitable or preferred in more challenging cases such as laparoscopic surgeries in obese patients.

Most of the patients in our study that underwent cystotomy repair had previous operations 41/68 (60.3\%). A multitude of factors are considered before a patient undergoes an operation and usually the benefit of the procedure outweighs the risks of surgical intervention. An attempt to minimize these risks, such as intraoperative complications, should be made but can never be eliminated. A significant surgical history, baseline comorbidities, chronic infections or inflammation, and complexity of the surgical case are some of the risk factors for complications. ${ }^{5,19}$ The most common complications during cystotomy repair include urinary leak, urinary tract infection, urinary retention, and stone formation. In our study, $16.2 \%$ of patients developed a complication. Although this seems high, our definition of complication was broad and may not have been directly related to the type of suture used during the bladder repair. Also, most of the complications were minor according to the Clavien-Dindo classification and did not result in further morbidity. In our study, the number of complications associated with barbed suture was not significantly different from braided suture. Similarly, a recent case series utilizing barbed suture for cystotomy repair did not result in additional complications. $^{2}$

This study has several limitations. With any study relying on electronic medical records, coding discrepancies could exist causing misclassification with data collection. Also, with limited post-operative follow-up, it is difficult to discern whether the surgical outcomes were long lasting. In our practice, we typically follow up with 
the patients 4-6 weeks after surgery and voiding cystometrogram or cystoscopy are not routinely performed. This database may not have included all observable patient characteristics that could confound the association between the use of barbed suture and patient outcome. Also, it could be argued that selection bias exists on account of a disproportionate number of patients were overweight or obese. Although this might account for some of the results seen within our study, the patient demographics within the state of West Virginia do not demonstrate substantial diversity with regard to weight. Furthermore, it is possible that non-modifiable patient factors could explain our observed results. Finally, since all the data was collected from a single academic, tertiary care hospital, this may limit the generalizability to nonacademic community hospitals.

\section{Conclusion}

Barbed suture may be comparable to standard braided suture for cystotomy repair. Therefore, barbed suture may offer a practical alternative to facilitate cystotomy repair in minimally invasive surgery, especially in patients with a high BMI. This study contributes to existing literature on treating urologic complications with a minimally invasive surgical approach. Further research is warranted to determine what, if any, long-term effects this technique will have.

\section{Abbreviations}

WVU, West Virginia University; BMI, body mass index.

\section{Data Sharing Statement}

The datasets used and/or analysed during the current study are available from the corresponding author on reasonable request.

\section{Ethics Approval and Consent to Participate}

This is a retrospective cohort study using the medical records from West Virginia Medicine Healthcare System. This study was approved by the West Virginia University Institutional Review Board (approval number 1909723019).

\section{Acknowledgments}

The abstract of this paper was presented at the 2021 Society for Laparoscopic Surgery/Minimally Invasive Surgery meeting as poster presentation. The poster's abstract was published in "Poster Abstracts" in the Journal of Minimally Invasive Gynecology, Volume 27, Issue 7, Supplement, November-December 2020, page 596.

\section{Author Contributions}

All authors made a significant contribution to the work reported, whether that is in the conception, study design, execution, acquisition of data, analysis and interpretation, or in all these areas; took part in drafting, revising or critically reviewing the article; gave final approval of the version to be published; have agreed on the journal to which the article has been submitted; and agree to be accountable for all aspects of the work.

\section{Funding}

Research reported in this publication was supported by the National Institute of General Medical Sciences of the National Institutes of Health under Award Number 2U54GM104942-02. The content is solely the responsibility of the authors and does not necessarily represent the official views of the National Institutes of Health. Authors RS, AS, SZ, MV, TT, and ODG have no other competing financial interests.

\section{Disclosure}

AS, MCV, TT, and ODG have no disclosures to report. RS reports non-financial support from Boston Scientific, outside of the submitted work. SZ reports non-financial support from Johnson and Johnson, outside of the submitted work. The authors report no other conflicts of interest in this work.

\section{References}

1. Lee JS, Choe JH, Lee HS, Seo JT. Urologic complications following obstetric and gynecologic surgery. Korean J Urol. 2012;53 (11):795-799. doi:10.4111/kju.2012.53.11.795

2. Glaser LM, Milad MP. Bowel and bladder injury repair and follow-up after gynecologic surgery. Obstet Gynecol. 2019;133(2):313-322. doi:10.1097/AOG.0000000000003067

3. Minas V, Gul N, Aust T, Rowlands D. Reducing the rate of abdominal hysterectomies: experience from a UK university teaching hospital. J Minim Invasive Gynecol. 2018;25(4):555-557. doi:10.1016/j. jmig.2017.11.002

4. Stany MP, Farley JH. Complications of gynecologic surgery. Surg Clin North Am. 2008;88(2):343-359, vii. doi:10.1016/j.suc.2007.12.004

5. Delacroix SE, Winters JC. Urinary tract injuries: recognition and management. Clin Colon Rectal Surg. 2010;23(03):221. doi:10.1055/ s-0030-1263063

6. Rao D, Yu H, Zhu H, Duan P. The diagnosis and treatment of iatrogenic ureteral and bladder injury caused by traditional gynaecology and obstetrics operation. Arch Gynecol Obstet. 2012;285(3):763-765. doi:10.1007/s00404-011-2075-7 
7. Figert PL, Park AE, Witzke DB, Schwartz RW. Transfer of training in acquiring laparoscopic skills. J Am Coll Surg. 2001;193(5):533-537. doi:10.1016/s1072-7515(01)01069-9

8. Schorge JO. Minimally invasive surgery in morbidly obese women. Obstet Gynecol. 2020;135(1):199-210. doi:10.1097/AOG.0000 000000003588

9. Chamsy D, King C, Lee T. The use of barbed suture for bladder and bowel repair. J Minim Invasive Gynecol. 2015;22(4):648-652. PMID: 25659867. doi:10.1016/j.jmig.2015.01.030

10. Dennis C, Sethu S, Nayak S, et al. Suture materials - current and emerging trends. J Biomed Mater Res A. 2016;104(6):1544-1559. doi:10.1002/jbm.a.35683

11. Yalcin S, Kibar Y, Tokas T, et al. In vivo comparison of "V-Loc 90 wound closure device" with "vicryl" and "monocryl" in regard to tissue reaction in a rabbit bladder model. Urology. 2018;116:231.e1231.e5. doi:10.1016/j.urology.2018.02.027

12. Dindo D, Demartines N, Clavien PA. Classification of surgical complications: a new proposal with evaluation in a cohort of 6336 patients and results of a survey. Ann Surg. 2004;240(2):205. doi:10.1097/01.sla.0000133083.54934.ae

13. Greenberg JA, Goldman RH. Barbed suture: a review of the technology and clinical uses in obstetrics and gynecology. Rev Obstet Gynecol. 2013;6:107-115.
14. Chamsy D, Lee T. The use of barbed suture in bladder and bowel surgery. Surg Technol Int. 2013;23:153-159.

15. Hagemann AR, McCourt CK, Varaday SS, Moore KN. Defining and mitigating the challenges of an older and obese population in minimally invasive gynecologic cancer surgery. Gynecol Oncol. 2018;148:601-608. doi:10.1016/j.ygyno.2017.12.020

16. Shah DK, Vitonis AF, Missmer SA. Association of body mass index and morbidity after abdominal, vaginal, and laparoscopic hysterectomy. Obstet Gynecol. 2015;125(3):589-598. doi:10.1097/ AOG.0000000000000698

17. Alessandri F, Remorgida V, Venturini PL, Ferrero S. Unidirectional barbed suture versus continuous suture with intracorporeal knots in laparoscopic myomectomy: a randomized study. J Minim Invasive Gynecol. 2010;17(6):725-729. doi:10.1016/j.jmig.2010.06.007

18. Angioli R, Plotti F, Montera R, et al. A new type of absorbable barbed suture for use in laparoscopic myomectomy. Int $J$ Gynaecol Obstet. 2012;117:220-223. doi:10.1016/j.ijgo.2011.12.023

19. Cohen AJ, Packiam VT, Nottingham CU, et al. Iatrogenic bladder injury: national analysis of 30-day outcomes. Urology. 2016:97:250-256. doi:10.1016/j.urology.2016.05.002

\section{Publish your work in this journal}

Research and Reports in Urology is an international, peer-reviewed, open access journal publishing original research, reports, editorials, reviews and commentaries on all aspects of adult and pediatric urology in the clinic and laboratory including the following topics: Pathology, pathophysiology of urological disease; Investigation and treatment of urological disease; Pharmacology of drugs used for the treatment of urological disease. The manuscript management system is completely online and includes a very quick and fair peer-review system, which is all easy to use. Visit http://www.dovepress.com/ testimonials.php to read real quotes from published authors. 